\title{
Patient Posture Monitoring System Based on Flexible Sensors
}

\author{
Youngsu Cha ${ }^{1, *}$, Kihyuk Nam ${ }^{1,2}$ and Doik Kim ${ }^{1}$ \\ 1 Center for Robotics Research, Korea Institute of Science and Technology, Seoul 02792, Korea; \\ todream623@naver.com (K.N.); doikkim@kist.re.kr (D.K.) \\ 2 Department of Mechanical Engineering, Hanyang University, Seoul 04763, Korea \\ * Correspondence: givemong@kist.re.kr; Tel.: +82-2-958-6949
}

Academic Editors: Hyun-Joong Chung and Tae-il Kim

Received: 9 February 2017; Accepted: 10 March 2017; Published: 13 March 2017

\begin{abstract}
Monitoring patients using vision cameras can cause privacy intrusion problems. In this paper, we propose a patient position monitoring system based on a patient cloth with unobtrusive sensors. We use flexible sensors based on polyvinylidene fluoride, which is a flexible piezoelectric material. The flexible sensors are inserted into parts close to the knee and hip of the loose patient cloth. We measure electrical signals from the sensors caused by the piezoelectric effect when the knee and hip in the cloth are bent. The measured sensor outputs are transferred to a computer via Bluetooth. We use a custom-made program to detect the position of the patient through a rule-based algorithm and the sensor outputs. The detectable postures are based on six human motions in and around a bed. The proposed system can detect the patient positions with a success rate over 88 percent for three patients.
\end{abstract}

Keywords: flexible sensor; patient cloth; piezoelectric material; real-time monitoring

\section{Introduction}

Recently, monitoring technologies geared to the care of patients and the elderly have received considerable attention [1-3]. Monitoring systems can be adapted for management applications in hospitals, nursing facilities, and the elderly that live alone [3-5]. These systems can be managed by only a few nurses and have great benefits, such as reducing healthcare cost and providing efficient service [6,7].

In this paper, we propose a patient posture monitoring system based on flexible sensors. The easiest way to monitor people is to use vision cameras [8]. It can be used for fall detection $[9,10]$, sleep monitoring [11,12], breathing detection [13,14], depression detection $[15,16]$, measuring vital signs $[17,18]$, and posture detection $[19,20]$. However, they can have blind spots because of the camera's position. In addition, vision-based monitoring systems have a privacy problem [8,21].

An alternative method is using sensing devices such as accelerometers and gyroscopes [22]. Without the invasion of user privacy, these devices can monitor human walking [23-25], posture transition [26-28], and daily activity [29-31]. Although the sensors protect privacy, detailed posture detection can be difficult. To collect sufficient data on the patient's position and posture, many sensors would be attached to the user. Further, the sensors have mostly rigid cases, which result in inconvenience to the users.

The feasibility of new monitoring systems using flexible sensors are considered as an option [32,33]. The flexible sensors can be developed by microfabrication process [34-38], textile weaving [39,40], or commercial materials [41]. The sensors are adapted for snoring detection system [41], mobility pattern research of a person on a bed [42], human motion sensing [43,44], and real-time health and fitness monitoring [45]. 
For our system, we use flexible piezoelectric sensors consisting of polyvinylidene fluoride (PVDF) [46-48], which is the one of the most flexible piezoelectric materials [49-51]. The piezoelectric sensors are installed into the parts close to the knee and hip of the loose patient cloth to be worn. When the knee and hip are bent, the sensors generate voltage signals that are measured by the analog-digital converter in a micro-controller module. The measured signals are wirelessly sent to a computer with a posture monitoring program. Therein, by the use of a rule-based algorithm and the processed sensor outputs, we detect six human motions occurring in and around the bed of three patients who are wearing the cloth.

From a practical point of view, this work addresses the untapped research question of sensing human motions from sensors embedded in a loose cloth. From a methodological point of view, the main contributions of this effort are: (i) developing a patient posture monitoring system using the loose patient cloth and flexible piezoelectric sensors; (ii) performing a thorough experimental campaign to assess the performance of the monitoring system; and (iii) conducting an analysis of the sensing characteristics of the flexible sensors in the given human motion conditions.

This paper is organized as follows. In Section 2, we introduce the proposed system setup with the description about the patient cloth, flexible sensor, and sensing module. In Section 3, we show and discuss the sensor outputs during the six transition motions between four positions. The decision method using the sensor outputs for the posture detection is reported in Section 4 . In Section 5, the patient posture monitoring system is experimentally demonstrated. The conclusions are summarized in the final section.

\section{System Setup}

We fabricate flexible sensors consisting of PVDF (produced by Measurement Specialties) and Mylar [47,48]. A PVDF layer with the size of $75 \times 25 \times 0.11 \mathrm{~mm}^{3}$ was attached to a Mylar sheet with $70 \times 25 \times 0.1 \mathrm{~mm}^{3}$ using 3M DP460 epoxy. The capacitance of the PVDF layer was $2.66 \mathrm{nF}$, measured using a FLUKE-17B + digital multimeter. To sense electrical signals from the PVDF, conductive adhesive $3 \mathrm{M}$ copper foil tape 1181 was utilized with copper electrodes on both sides. Two wires were soldered to access the copper electrodes, and were connected to a sensing module. Each component in the sensing module was soldered on a universal printed circuit board (PCB) with the size of $50 \times 50 \mathrm{~mm}^{2}$. Figure 1 displays the fabricated flexible sensor and sensing module.

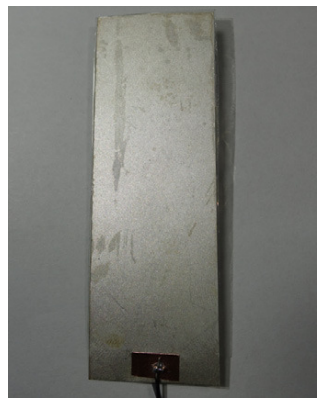

(a)

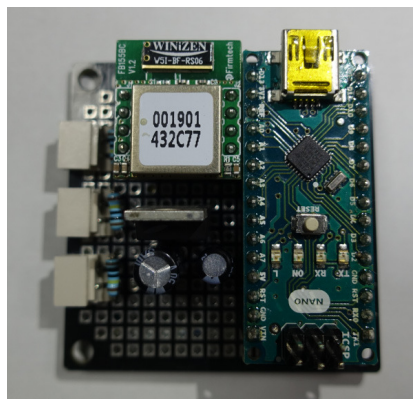

(b)

Figure 1. Pictures of the (a) flexible sensor and (b) sensing module.

The sensing module was utilized to record the voltages from the sensors and to transfer the data wirelessly. The voltage signals were measured through 10-bit analog-digital converters of an Arduino Nano micro-controller, with load resistors, $R=1 \mathrm{M} \Omega$ (see Figure 2). One end of the load resistor was connected to a $2.5 \mathrm{~V}$ source generated by a SPX1587AU-2.5 regulator. This connection makes the sensor outputs maintain a positive value, which can be detected in the analog-digital converters. Ideally, the sensor output at $2.5 \mathrm{~V}$ is 512 (binary number 1000000000). The digitalized sensor outputs and measured time are transferred to a computer through a FB155BC Bluetooth unit. The sensor outputs 
are recoded and sent at $90 \sim 100 \mathrm{~Hz}$. The sensing module is supported by a lithium polymer battery with the voltage output of $7.4 \mathrm{~V}$ and a capacity of $650 \mathrm{mAh}$.

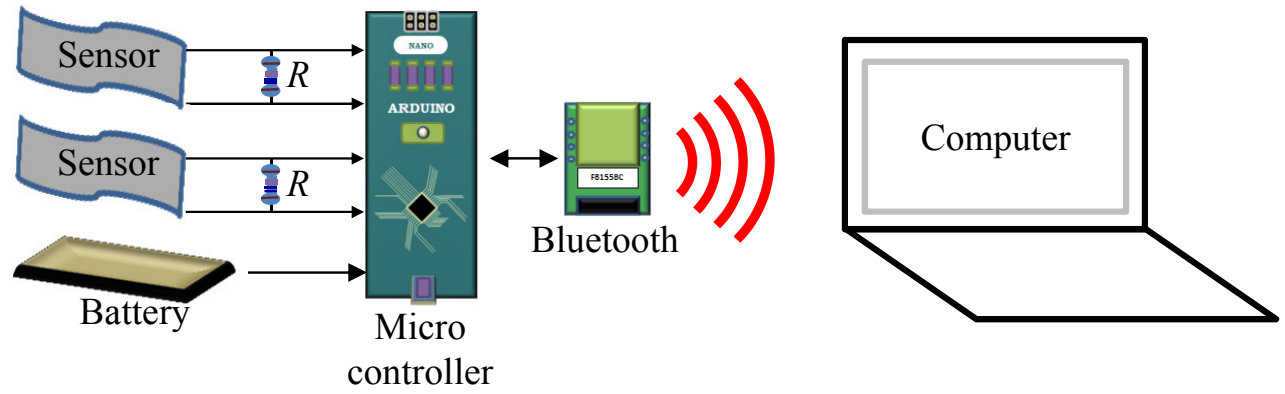

Figure 2. Schematic of the sensing system consisting of two flexible sensors and one sensing module.

The sensing system was installed in a patient cloth. In particular, two flexible sensors were attached to two parts of the patient pants using pockets, as shown in Figure 3a. The pockets were positioned in the left knee and hip parts. Figure $3 b$ displays a male student (Patient1) wearing the patient cloth. The sensing module was put into a vest pocket of the patient's upper garment.

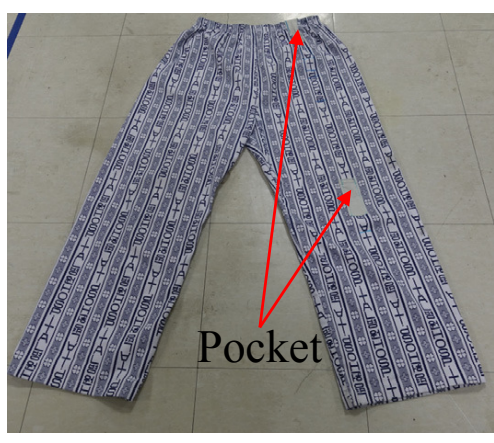

(a)

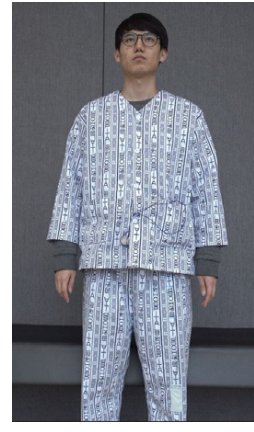

(b)

Figure 3. Pictures of the (a) patient pants with two pockets for the flexible sensors and (b) patient wearing the sensing system.

\section{Patient Posture and Sensor Output}

We focus on the four positions of a patient in and around a bed. In particular, they are standing, sitting, sitting knee extension, and supine poses, as shown in Figure 4. The standing pose is when a patient is in a vertical position. The sitting position means that a patient sits with bent knees on a bed. The sitting knee extension posture is to sit with the knees outstretched on a bed. The supine pose is to lie horizontally on a bed. In addition, we show the shapes of the sensors as each pose in the inset of Figure 4.

Figure 5 displays the time traces of the sensor output voltages at the knee and hip during the six transitions between the four positions. Specifically, when the transition from the standing to sitting position occurs, the sensors at the knee and hip are bent along with the joints. Interestingly, the phase of the sensor outputs are opposite because of the difference of each bending direction (see Figure 4a). Further, the hip value is higher than the knee; that is, the bending at the hip is larger. When the pose of the patient comes back to the standing position, the sensor outputs in Figure $5 \mathrm{~b}$ are the counter images of Figure $5 a$. Figure $5 c$ displays the signals when the knee is extended while sitting. This signal at the knee has mostly negative values, whereas the one at the hip oscillates between positive and negative values. In Figure 5d, we observe the reverse phase of that case from the sitting knee extension to the bent knee position. Figure 5e,f display the positive and negative values from only the hip sensor by 
the extension and bending of the joint. In addition, we observe that the pose transitions happen for approximately $2 \mathrm{~s}$.

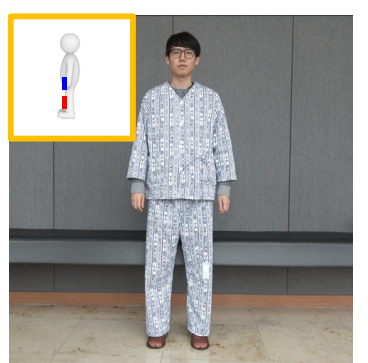

(a)

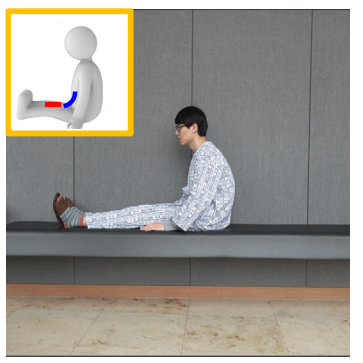

(c)

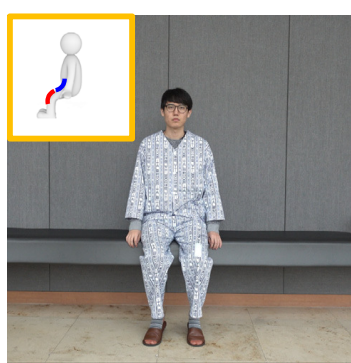

(b)

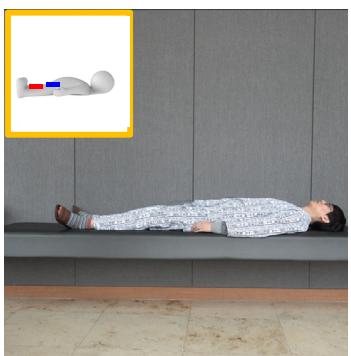

(d)

Figure 4. Patient postures with the (a) standing; (b) sitting; (c) sitting knee extension; and (d) supine positions. In the inset, the red and blue lines are the sensor shapes at the knee and hip, respectively.

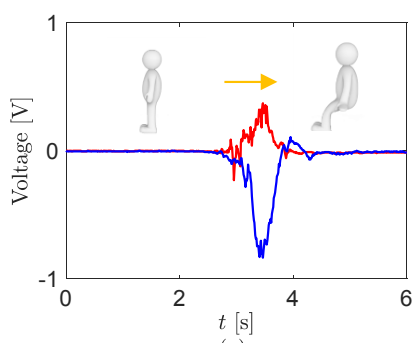

(a)

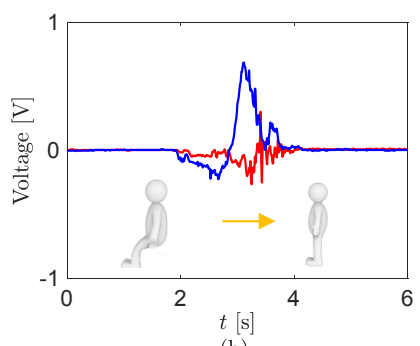

(b)

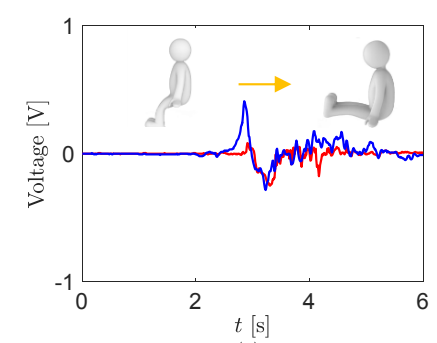

(c)

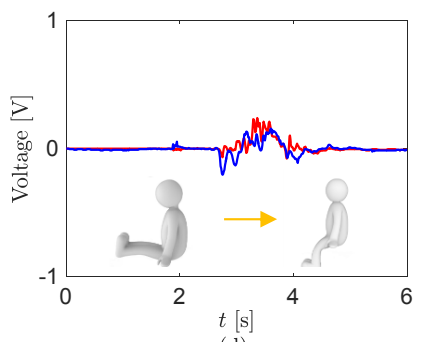

(d)

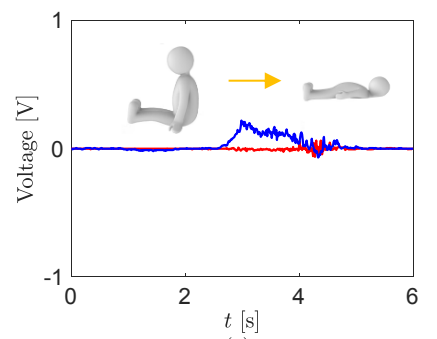

(e)

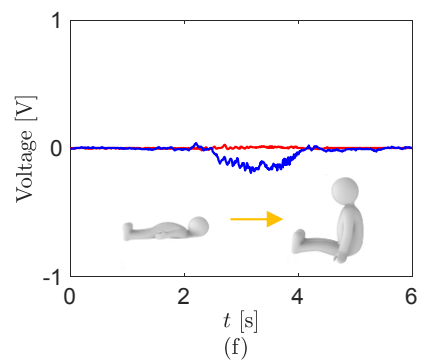

Figure 5. Digitalized sensor outputs at the transition (a) from the standing to sitting position; (b) from the sitting to standing position; (c) from the sitting to sitting knee extension position; (d) from the sitting knee extension to sitting position; (e) from the sitting knee extension to supine position; and (f) from the supine to sitting knee extension position. The red and blue lines are the data of the sensor at the knee and hip, respectively.

\section{Decision Method}

To detect the patient's postures from the sensor outputs in Figure 5, we do data processing by using C\# language. The main processing steps are described as follows. 
Step 1: The offsets of the digitalized sensor outputs in Figure 5 have removed. We use the average value of 9000 in the dataset as the offset. It is mathematically described as

$$
y_{\mathrm{n}}=x_{\mathrm{n}}-\frac{\sum_{\mathrm{m}=\mathrm{n}-8999}^{\mathrm{n}} x_{\mathrm{m}}}{9000}
$$

where $x_{\mathrm{n}}$ is the digitalized sensor output, and $y_{\mathrm{n}}$ is the value without the offset.

Step 2: The values after removing the offset are integrated as the time

$$
z_{\mathrm{n}}=z_{\mathrm{n}-1}+y_{\mathrm{n}} \times \Delta t_{\mathrm{n}}
$$

where $z_{\mathrm{n}}$ is the integrated value and $\Delta t_{\mathrm{n}}$ is the time interval.

The processed values from the sensor outputs in Figure 5 are shown in Figure 6. In Figure 6a, the positive knee value and the negative hip value are saturated. Further, they have the opposite values in Figure $6 \mathrm{~b}$. This is due to the characteristics of the piezoelectric sensor outputs, of which raw voltage and integration at a load resistance may be proportional to the angular velocity and angle, respectively [52]. The profiles of the processed values from the knee in Figure 6c,d are the same as in Figure 6a,b, respectively. The values at the hip in Figure $6 c, d$ are oscillated and converged to much smaller ones than in Figure 6a,b. In Figure $6 \mathrm{e}, \mathrm{f}$, while the values at the knee are not moving, the ones at the hip are saturated to the larger positive and negative values, respectively. The converged values at the hip are slightly more decreased than in Figure 6a,b. Reasons for the decreases may be ascribed to the changes of the bending of the pants during the turning motion between the sitting position and sitting knee extension position.

Step 3: We use a rule-based algorithm $[53,54]$ to decide the postures of the patient wearing the cloth with the sensors. Specifically, the bending and extension of the knee and hip at $90^{\circ}$ of the sensors in the patient's pants can be detected from the processed values in Figure 6. We compare the difference between the current processed value and the previous one before 200 counts; that is,

$$
\begin{gathered}
D_{\mathrm{n}}=z_{\mathrm{n}}-z_{\mathrm{n}-199} \\
\left\{\begin{array}{cc}
D_{\mathrm{n}}^{\text {knee }}>C^{\text {knee }} & \text { knee bending } \\
\left|D_{\mathrm{n}}^{\text {knee }}\right|<C^{\text {knee }} & - \\
D_{\mathrm{n}}^{\text {knee }}<-C^{\text {knee }} & \text { knee extension }
\end{array}\right. \\
\left\{\begin{array}{cc}
D_{\mathrm{n}}^{\text {hip }}>C^{\text {hip }} & \text { hip extension } \\
\left|D_{\mathrm{n}}^{\text {hip }}\right|<C^{\text {hip }} & - \\
D_{\mathrm{n}}^{\text {hip }}<-C^{\text {hip }} & \text { hip bending }
\end{array}\right.
\end{gathered}
$$

where $D_{\mathrm{n}}^{\text {knee }}$ and $D_{\mathrm{n}}^{\text {hip }}$ are the differences at the knee and hip, respectively. $C^{\text {knee }}$ and $C^{\text {hip }}$ are the decision values at the knee and hip, respectively.

The decision values for the detection are selected as $C^{\text {knee }}=7.5$ and $C^{\text {hip }}=20$ by preliminary tests with each having 100 repetitions between the standing and the sitting positions; see the histogram and statistical data in Figure 7. The decision values are $C^{\text {knee } /}-C^{\text {knee }}$ and

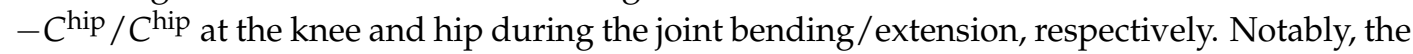
decision values and signs at the knee and hip are different. The value gap can be attributed to the effect of each joint structure, which results in different mechanical displacement of the sensor. The variations under the decision values expect that the bending and extension of the 
joints are smaller than $90^{\circ}$. The opposite sign results from the different bending direction at the knee and hip. The decision values may differ as the body structure of the patient varies.

Step 4: By using the previous posture state and the decisions in Step 3, we predict the current position of the patient; that is,

$$
S_{\mathrm{n}}=f\left(D_{\mathrm{n}}^{\text {knee }}, D_{\mathrm{n}}^{\text {hip }}, S_{\mathrm{n}-1}\right)
$$

where $S_{\mathrm{n}}$ is the posture state and $f(\cdot)$ is the decision function.

Figure 8 displays the flow chart to decide the position of the patient. For example, when $D_{n}^{\text {knee }}$ is under $-C^{\text {knee }}$ and $D_{\mathrm{n}}^{\text {hip }}$ is higher than $C^{\text {hip }}$ at the sitting pose, the transferred pose is standing. In the program, we use the standing position as the initial state.

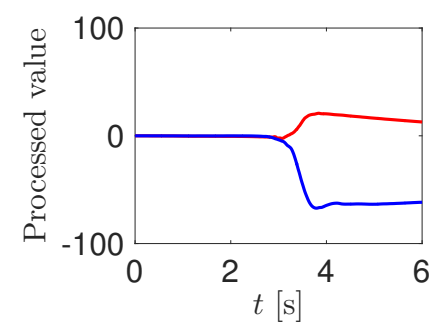

(a)

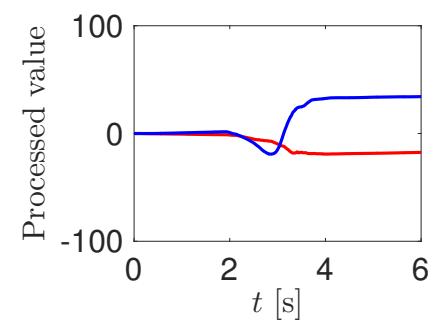

(b)

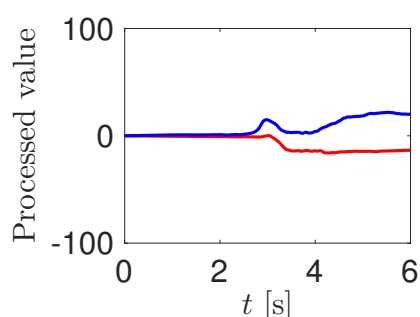

(c)

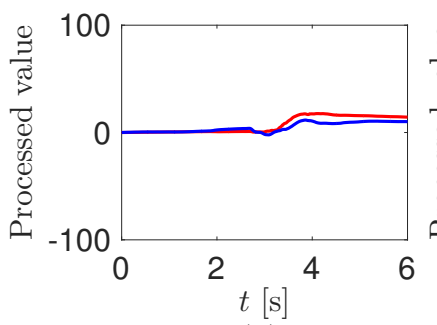

(d)

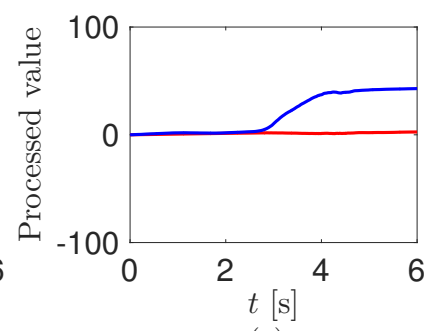

(e)

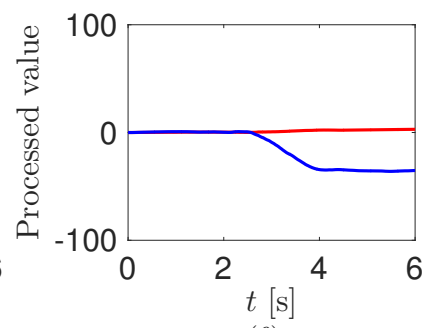

(f)

Figure 6. Signal processed values from the sensor outputs at the transition (a) from the standing to sitting position; (b) from the sitting to standing position; (c) from the sitting to sitting knee extension position; (d) from the sitting knee extension to sitting position; (e) from the sitting knee extension to supine position; and (f) from the supine to sitting knee extension position. The red and blue lines are the data of the sensor at the knee and hip, respectively.

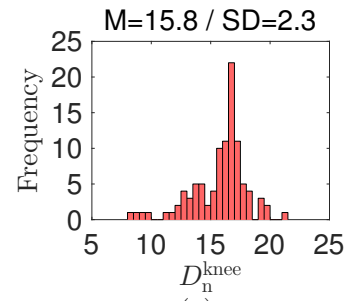

(a)

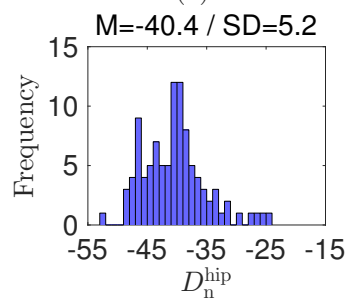

(b)

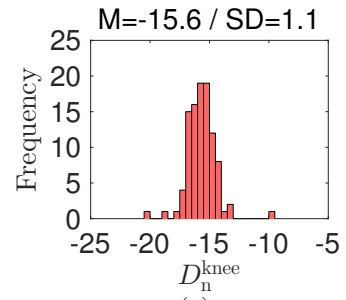

(c)

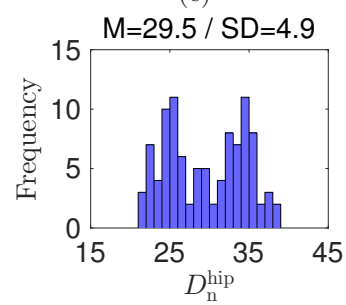

(d)

Figure 7. Histograms of the difference $D_{\mathrm{n}}$. (a) $D_{\mathrm{n}}^{\text {knee }}$ and (b) $D_{\mathrm{n}}^{\text {hip }}$ at the transition from the standing to sitting. (c) $D_{\mathrm{n}}^{\text {knee }}$ and (d) $D_{\mathrm{n}}^{\text {hip }}$ at the transition from the sitting to standing. "M" and "SD" indicate their mean and standard deviation. 


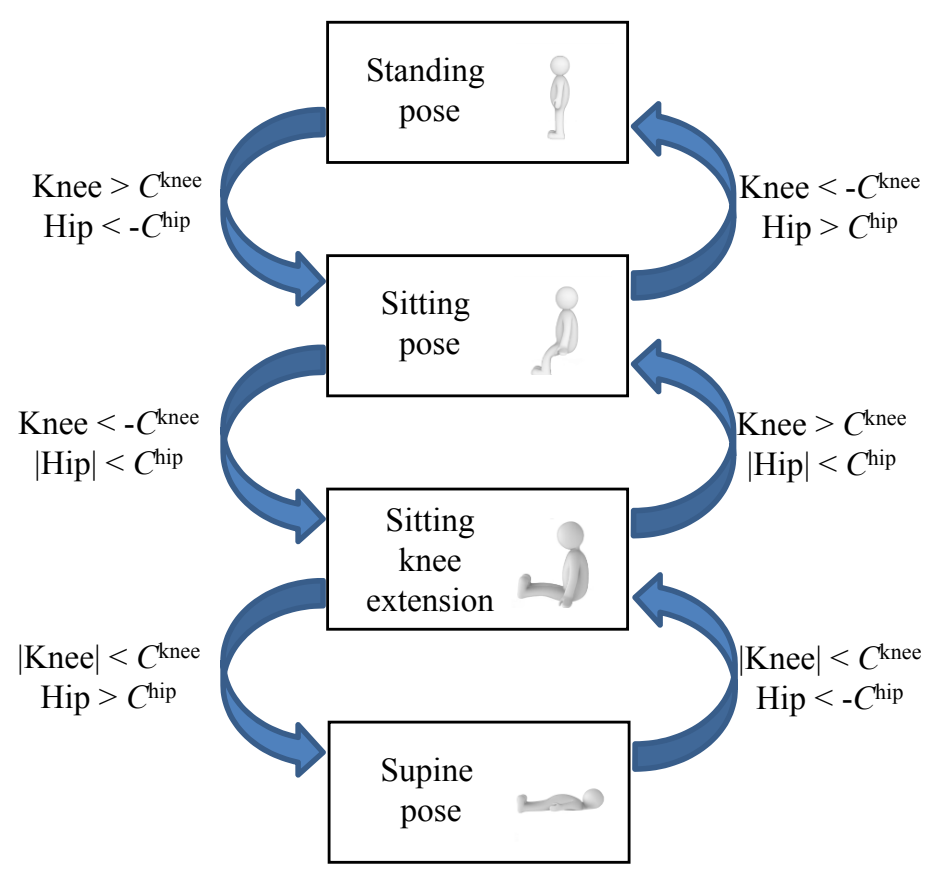

Figure 8. Flow chart for posture decisions.

\section{System Operation}

In this section, we demonstrate the operation of the patient posture monitoring system. The student wearing the patient cloth with the sensors goes through the six motions (i) from the standing to sitting position; (ii) from the sitting to standing position; (iii) from the sitting to sitting knee extension position; (iv) from the sitting knee extension to sitting position; (v) from the sitting knee extension to supine position; and (vi) from the supine to sitting knee extension position. Figure 9 displays the computer screen of the patient posture monitoring system for the six transitions. The green borders show the current postures of the patient. The left and right bottom graphs in Figure 9 display the processed values at the knee and hip, respectively. The operation of the patient posture monitoring system is displayed in Video S1 as a supplementary material.

To assess the patient posture monitoring system, a total of 900 experiments from three students wearing the patient clothes with different size (50 per each motion and person) were performed. We use the decision values for Patient2, $C^{\text {knee }}=10$ and $C^{\text {hip }}=20$, and Patient $3, C^{\text {knee }}=7.5$ and $C^{\text {hip }}=10$, selected by preliminary tests. Table 1 shows the success rate of the six transition motions of the three patients. All motions of three patients were detected with a success rate over $88 \%$. A few times, we failed to detect the motions because of low sensor values. In motion cases (i) and (ii), the position of the knee sensor during bending or releasing was sometimes misaligned. In motion cases (iii) and (iv), this may be ascribed to the release of the hip sensor for turning motion between the sitting and sitting knee extension positions. In motion cases (v) and (vi), the hip sensor could have a low value because of the subsequent release of the bent hip sensor and misalignment. 


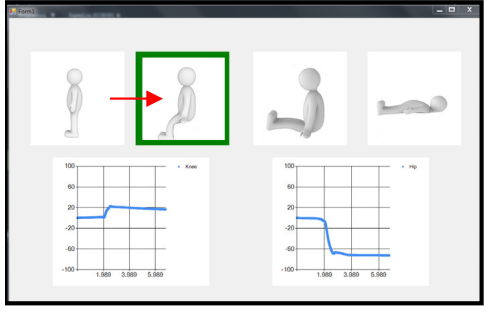

(a)

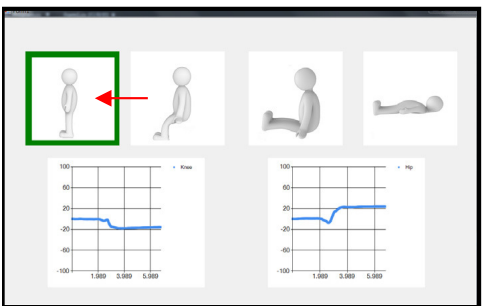

(b)

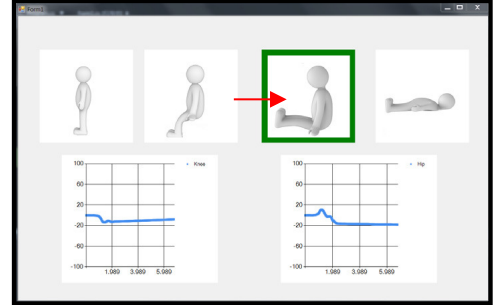

(c)

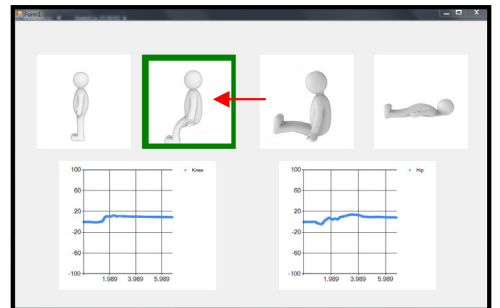

(d)

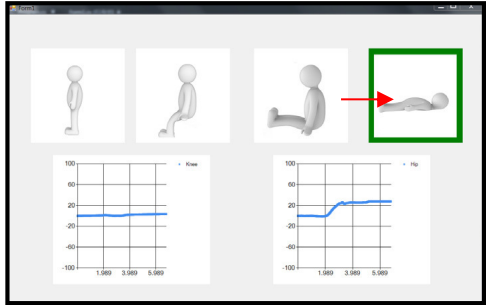

(e)

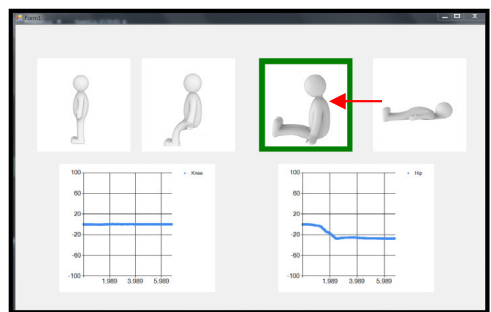

(f)

Figure 9. Snapshots of the computer screen showing the estimated patient postures and signal processed values from the sensor outputs at the transition (a) from the standing to sitting position (i); (b) from the sitting to standing position (ii); (c) from the sitting to sitting knee extension position (iii); (d) from the sitting knee extension to sitting position (iv); (e) from the sitting knee extension to supine position (v); and (f) from the supine to sitting knee extension position (vi). The arrows are added to represent the transitions from the previous positions.

Table 1. Success rate of the patient posture monitoring system.

\begin{tabular}{ccccccc}
\hline Motion & (i) & (ii) & (iii) & (iv) & (v) & (vi) \\
\hline Patient1 (M-23 years-185 cm-72 kg) $(\%)$ & 100 & 100 & 92 & 96 & 90 & 100 \\
Patient2 (M-24 years-171 cm-67 kg) $(\%)$ & 88 & 96 & 96 & 98 & 94 & 98 \\
Patient3 (F-21 years-160 cm-51 kg) $(\%)$ & 98 & 96 & 88 & 94 & 100 & 100 \\
\hline
\end{tabular}

\section{Conclusions}

In this paper, we demonstrated a patient posture monitoring system based on a patient cloth with embedded flexible piezoelectric sensors. The patient cloth was fitted loosely, and the flexible sensors were positioned in the knee and hip parts of the cloth. The sensor generated electrical responses corresponding to the bending and extension of each joint. The sensor outputs were wirelessly transferred to a computer with a custom-made program. The data accurately predicted the position of the patient through a rule-based algorithm after processing the electrical signals. We tested the monitoring system with six motions between four positions that can happen in or around a bed. Our system operation was working well with a success rate over $88 \%$ for three patients. The demonstration of the system suggests a patient monitoring system using unobtrusive wearable sensors. Without a vision camera, this monitoring system can monitor the position of patients while protecting their privacy. This has beneficial uses in hospitals and nursing facilities. Future studies will focus on the performance evaluation of the system through adaptation in various test subject groups and comparison with other devices.

Supplementary Materials: The following are available online at http:/ /www.mdpi.com/1424-8220/17/3/584/s1, Video S1: Operation of the patient posture monitoring system.

Acknowledgments: This work was supported by the KIST flagship program (Project No. 2E27200). The authors would like to thank Hojoon Kim and Jiyeon Seo for their help with the experiments.

Author Contributions: Youngsu Cha led the experiment, performed data analysis, and drafted the manuscript. Kihyuk Nam conducted the experiment. Doik Kim contributed to the setup of the experiment. 
Conflicts of Interest: The authors declare no conflict of interest.

\section{References}

1. Pawar, P.; Jones, V.; Van Beijnum, B.F.; Hermens, H. A framework for the comparison of mobile patient monitoring systems. J. Biomed. Inf. 2012, 45, 544-556.

2. Liang, T.; Yuan, Y.J. Wearable medical monitoring systems based on wireless networks: A review. IEEE Sens. J. 2016, 16, 8186-8199.

3. Peetoom, K.K.B.; Lexis, M.A.S.; Joore, M.; Dirksen, C.D.; De Witte, L.P. Literature review on monitoring technologies and their outcomes in independently living elderly people. Disabil. Rehabil. Assist. Technol. 2015, 10, 271-294.

4. Chen, S.; Kao, T.; Chan, C.; Huang, C.; Chiang, C.; Lai, C.; Tung, T.; Wang, P. A reliable transmission protocol for zigbee-based wireless patient monitoring. IEEE Trans. Inf. Technol. Biomed. 2012, 16, 6-16.

5. Baig, M.M.; Gholamhosseini, H. Smart health monitoring systems: An overview of design and modeling. J. Med. Syst. 2013, 37, 1-14.

6. Varshney, U. Pervasive healthcare and wireless health monitoring. Mob. Netw. Appl. 2007, 12, 113-127.

7. Sneha, S.; Varshney, U. Enabling ubiquitous patient monitoring: Model, decision protocols, opportunities and challenges. Decis. Support Syst. 2009, 46, 606-619.

8. Sathyanarayana, S.; Satzoda, R.K.; Sathyanarayana, S.; Thambipillai, S. Vision-based patient monitoring: A comprehensive review of algorithms and technologies. J. Ambient Intell. Humaniz. Comput. 2015, doi:10.1007/s12652-015-0328-1.

9. Yu, M.; Rhuma, A.; Naqvi, S.M.; Wang, L.; Chambers, J. A posture recognition-based fall detection system for monitoring an elderly person in a smart home environment. IEEE Trans. Inf. Technol. Biomed. 2012, $16,1274-1286$.

10. Mubashir, M.; Shao, L.; Seed, L. A survey on fall detection: Principles and approaches. Neurocomputing 2013, 100, 144-152.

11. Malakuti, K.; Albu, A.B. Towards an intelligent bed sensor: Non-intrusive monitoring of sleep irregularities with computer vision techniques. In Proceedings of the 20th International Conference on Pattern Recognition, Istanbul, Turkey, 23-26 August 2010; pp. 4004-4007.

12. Chen, L.C.; Chen, K.; Hung, Y. A sleep monitoring system based on audio, video and depth information for detecting sleep events. In Proceedings of the IEEE International Conference on Multimedia and Expo, Chengdu, China, 14-18 July 2014; pp. 1-6.

13. Kuo, Y.; Lee, J.; Chung, P. A visual context-awareness-based sleeping-respiration measurement system. IEEE Trans. Inf. Technol. Biomed. 2010, 14, 255-265.

14. Martinez, M.; Stiefelhagen, R. Breath rate monitoring during sleep using near-IR imagery and PCA. In Proceedings of the 21st International Conference on Pattern Recognition, Tsukuba, Japan, 11-15 November 2012; pp. 3472-3475.

15. Cohn, J.F.; Kruez, T.S.; Matthews, I.; Yang, Y.; Nguyen, M.H.; Padilla, M.T.; Zhou, F.; De la Torre, F. Detecting depression from facial actions and vocal prosody. In Proceedings of the 3rd International Conference on Affective Computing and Intelligent Interaction and Workshops, Amsterdam, The Netherlands, 10-12 September 2009; pp. 1-7.

16. Alghowinem, S.; Goecke, R.; Wagner, M.; Parker, G.; Breakspear, M. Eye movement analysis for depression detection. In Proceedings of the IEEE International Conference on Image Processing, Melbourne, Australia, 15-18 September 2013; pp. 4220-4224.

17. Poh, M.; McDuff, D.J.; Picard, R.W. Non-contact, automated cardiac pulse measurements using video imaging and blind source separation. Opt. Express 2010, 18, 10762-10774.

18. Kwon, S.; Kim, H.; Park, K.S. Validation of heart rate extraction using video imaging on a built-in camera system of a smartphone. In Proceedings of the Annual International Conference of the IEEE Engineering in Medicine and Biology Society, San Diego, CA, USA, 28 August-1 September 2012; pp. 2174-2177.

19. Brulin, D.; Benezeth, Y.; Courtial, E. Posture recognition based on fuzzy logic for home monitoring of the elderly. IEEE Trans. Inf. Technol. Biomed. 2012, 16, 974-982. 
20. Obdržálek, S.; Kurillo, G.; Ofli, F.; Bajcsy, R.; Seto, E.; Jimison, H.; Pavel, M. Accuracy and robustness of Kinect pose estimation in the context of coaching of elderly population. In Proceedings of the Annual International Conference of the IEEE Engineering in Medicine and Biology Society, San Diego, CA, USA, 28 August-1 September 2012; pp. 1188-1193.

21. Igual, R.; Medrano, C.; Plaza, I. Challenges, issues and trends in fall detection systems. Biomed. Eng. Online 2013, 12, 1 .

22. Bulling, A.; Blanke, U.; Schiele, B. A tutorial on human activity recognition using body-worn inertial sensors. ACM Comput. Surv. 2014, 46, 33.

23. Miyazaki, S. Long-term unrestrained measurement of stride length and walking velocity utilizing a piezoelectric gyroscope. IEEE Trans. Biomed. Eng. 1997, 44, 753-759.

24. Morris, S.J.; Paradiso, J.A. A compact wearable sensor package for clinical gait monitoring. Offspring 2003, 1, 7-15.

25. Zhang, R.; Hoflinger, F.; Reindl, L. Inertial sensor based indoor localization and monitoring system for emergency responders. IEEE Sens. J. 2013, 13, 838-848.

26. Ayrulu-Erdem, B.; Barshan, B. Leg motion classification with artificial neural networks using wavelet-based features of gyroscope signals. Sensors 2011, 11, 1721-1743.

27. Regterschot, G.R.H.; Folkersma, M.; Zhang, W.; Baldus, H.; Stevens, M.; Zijlstra, W. Sensitivity of sensor-based sit-to-stand peak power to the effects of training leg strength, leg power and balance in older adults. Gait Posture 2014, 39, 303-307.

28. Regterschot, G.R.H.; Zhang, W.; Baldus, H.; Stevens, M.; Zijlstra, W. Accuracy and concurrent validity of a sensor-based analysis of sit-to-stand movements in older adults. Gait Posture 2016, 45, 198-203.

29. Veltink, P.H.; Bussmann, H.B.J.; De Vries, W.; Martens, W.J.; Van Lummel, R.C. Detection of static and dynamic activities using uniaxial accelerometers. IEEE Trans. Rehabil. Eng. 1996, 4, 375-385.

30. Najafi, B.; Aminian, K.; Paraschiv-Ionescu, A.; Loew, F.; Bula, C.J.; Robert, P. Ambulatory system for human motion analysis using a kinematic sensor: Monitoring of daily physical activity in the elderly. IEEE Trans. Biomed. Eng. 2003, 50, 711-723.

31. Brodie, M.A.D.; Coppens, M.J.M.; Lord, S.R.; Lovell, N.H.; Gschwind, Y.J.; Redmond, S.J.; Del Rosario, M.B.; Wang, K.; Sturnieks, D.L.; Persiani, M.; et al. Wearable pendant device monitoring using new wavelet-based methods shows daily life and laboratory gaits are different. Med. Biol. Eng. Comput. 2016, 54, 663-674.

32. Rogers, J.A.; Someya, T.; Huang, Y. Materials and mechanics for stretchable electronics. Science 2010, 327, 1603-1607.

33. Rogers, J.A. Electronics for the human body. J. Am. Med. Assoc. 2015, 313, 561-562.

34. Zhou, J.; Gu, Y.; Fei, P.; Mai, W.; Gao, Y.; Yang, R.; Bao, G.; Wang, Z.L. Flexible piezotronic strain sensor. Nano Lett. 2008, 8, 3035-3040.

35. Segev-Bar, M.; Haick, H. Flexible sensors based on nanoparticles. ACS Nano 2013, 7, 8366-8378.

36. Persano, L.; Dagdeviren, C.; Su, Y.; Zhang, Y.; Girardo, S.; Pisignano, D.; Huang, Y.; Rogers, J.A. High performance piezoelectric devices based on aligned arrays of nanofibers of poly(vinylidenefluorideco-trifluoroethylene). Nat. Commun. 2013, 4, 1633.

37. Liu, Y.; Norton, J.S.; Qazi, R.; Zou, Z.; Ammann, K.R.; Liu, H.; Yan, L.; Tran, P.L.; Jang, K.I.; Lee, J.W.; et al. Epidermal mechano-acoustic sensing electronics for cardiovascular diagnostics and human-machine interfaces. Sci. Adv. 2016, 2, e1601185.

38. Vilela, D.; Romeo, A.; Sánchez, S. Flexible sensors for biomedical technology. Lab Chip 2016, 16, $402-408$.

39. Cherenack, K.; Zysset, C.; Kinkeldei, T.; Münzenrieder, N.; Tröster, G. Woven electronic fibers with sensing and display functions for smart textiles. Adv. Mater. 2010, 22, 5178-5182.

40. Trindade, I.G.; Machado da Silva, J.; Miguel, R.; Pereira, M.; Lucas, J.; Oliveira, L.; Valentim, B.; Barreto, J.; Santos Silva, M. Design and Evaluation of Novel Textile Wearable Systems for the Surveillance of Vital Signals. Sensors 2016, 16, 1573.

41. Hwang, S.; Han, C.M.; Yoon, H.N.; Lee, Y.J.; Jeong, D.; Park, K.S. Polyvinylidene fluoride sensor-based method for unconstrained snoring detection. Physiol. Meas. 2015, 36, 1399.

42. Cho, S.H.; Cho, S. A Noninvasive Sensor System for Discriminating Mobility Pattern on a Bed. In Information Science and Applications; Springer: Berlin/Heidelberg, Germany, 2015; pp. 35-42.

43. Ryu, S.; Lee, P.; Chou, J.B.; Xu, R.; Zhao, R.; Hart, A.J.; Kim, S.G. Extremely elastic wearable carbon nanotube fiber strain sensor for monitoring of human motion. ACS Nano 2015, 9, 5929-5936. 
44. Tajitsu, Y. Sensing complicated motion of human body using piezoelectric chiral polymer fiber. Ferroelectrics 2015, 480, 32-38.

45. Imani, S.; Bandodkar, A.J.; Mohan, A.M.V.; Kumar, R.; Yu, S.; Wang, J.; Mercier, P.P. A wearable chemical-electrophysiological hybrid biosensing system for real-time health and fitness monitoring. Nat. Commun. 2016, 7, 11650.

46. Broadhurst, M.G.; Davis, G.T.; McKinney, J.E.; Collins, R.E. Piezoelectricity and pyroelectricity in polyvinylidene fluoride-A model. J. Appl. Phys. 1978, 49, 4992-4997.

47. Akaydin, H.D.; Elvin, N.; Andreopoulos, Y. Energy harvesting from highly unsteady fluid flows using [iezoelectric materials]. J. Intell. Mater. Syst. Struct. 2010, 21, 1263-1278.

48. Cha, Y.; Hong, J.; Lee, J.; Park, J.M.; Kim, K. Flexible piezoelectric energy harvesting from mouse click motions. Sensors 2016, 16, 1045.

49. Shen, D. Piezoelectric Energy Harvesting Devices for Low Frequency Vibration Applications; ProQuest: Ann Arbor, MI, USA, 2009.

50. Farinholt, K.M.; Pedrazas, N.A.; Schluneker, D.M.; Burt, D.W.; Farrar, C.R. An energy harvesting comparison of piezoelectric and ionically conductive polymers. J. Intell. Mater. Syst. Struct. 2009, 20, 633-642.

51. Tiwari, R.; Kim, K.J. IPMC as a mechanoelectric energy harvester: Tailored properties. Smart Mater. Struct. 2013, 22, 015017.

52. Cha, Y.; Hong, S. Energy harvesting from walking motion of a humanoid robot using a piezoelectric composite. Smart Mater. Struct. 2016, 25, 10 LT01.

53. Nadler, M.; Smith, E.P. Pattern Recognition Engineering; Wiley-Interscience: NewYork, NY, USA, 1993.

54. Tunçel, O.; Altun, K.; Barshan, B. Classifying human leg motions with uniaxial piezoelectric gyroscopes. Sensors 2009, 9, 8508-8546.

(C) 2017 by the authors. Licensee MDPI, Basel, Switzerland. This article is an open access article distributed under the terms and conditions of the Creative Commons Attribution (CC BY) license (http:/ / creativecommons.org/licenses/by/4.0/). 